\title{
AVALIAÇÃO DE PROJETOS EM FEIRAS CIENTÍFICAS ESTUDANTIS COMBINANDO VALUE-FOCUSED THINKING E ANALYTIC HIERARCHY PROCESS
}

\author{
Rafael Verão Françozo \\ Universidade Federal de São Paulo (UNIFESP) - Instituto Tecnológico de \\ Aeronáutica (ITA) \\ Pça Mal. E. Gomes, 50, Vila das Acácias, CEP 12228-900, São José dos Campos \\ rvfrancozo@gmail.com
}

\section{Amanda Cecília Simões da Silva}

Instituto Tecnológico de Aeronáutica (ITA)

Pça Mal. E. Gomes, 50, Vila das Acácias, CEP 12228-900, São José dos Campos amandaac@ita.br

Mischel Carmen N. Belderrain

Instituto Tecnológico de Aeronáutica (ITA)

Pça Mal. E. Gomes, 50, Vila das Acácias, CEP 12228-900, São José dos Campos carmen@ita.br

\section{Resumo}

Feiras estudantis são bastante populares e um dos principais eventos realizados no Instituto Federal de Educação, Ciência e Tecnologia de Mato Grosso do Sul (IFMS) durante a Semana Nacional de Ciência e Tecnologia. Este estudo tem por objetivo apresentar um modelo de avaliação dos projetos das feiras estudantis no âmbito do IFMS considerando aspectos subjetivos no julgamento dos avaliadores. Para este propósito foi adotada uma abordagem envolvendo Value-Focused Thinking (VFT) para identificação dos objetivos e critérios e Analytic Hierarchy Process (AHP) para avaliação. Os resultados indicam julgamentos mais consistentes considerando a subjetividade dos avaliadores com relação aos critérios. A abordagem proposta envolvendo VFT e AHP contribuiu com a definição dos critérios a serem avaliados assim como o grau de importância de cada critério para a avaliação geral dos projetos.

Palavras-chave: Problema de Decisão Multicritério, VFT, AHP, Ratings, Projetos. 


\section{Abstract}

Student fairs are very popular and one of the main events held at the Federal Institute of Education, Science and Technology of Mato Grosso do Sul (IFMS) during the National Week of Science and Technology. The objective of this study is to present a model for evaluating the projects of student fairs within the scope of the IFMS, considering subjective aspects in the judgment of the evaluators. For this purpose it was adopted an approach involving Value-Focused Thinking (VFT) to identify the objectives and criteria and Analytic Hierarchy Process (AHP) for evaluation. The results indicate more consistent judgments considering the subjectivity of the evaluators in relation to the criteria. The proposed approach involving VFT and AHP contributed to the definition of the criteria to be evaluated as well as the degree of importance of each criterion for the overall evaluation of projects.

Keywords: Multicriteria Decision Problem, VFT, AHP, Ratings, Projects.

\section{Como Citar:}

FRANÇOZO, R.V.; SILVA, A. C. S.; BELDERRAIN, M. C. N. Avaliação de Projetos em Feiras Científicas Estudantis Combinando Value-Focused Thinking e Analytic Hierarchy Process. In: SIMPÓSIO DE PESQUISA OPERACIONAL E LOGÍSTICA DA MARINHA, 19., 2019, Rio de Janeiro, RJ. Anais [...]. Rio de Janeiro: Centro de Análises de Sistemas Navais, 2019.

\section{INTRODUÇÃO}

Feiras, mostras e exposições científicas estudantis são importantes instrumentos para promoção da ciência, formação de hábitos, habilidades e de atitudes científicas de estudantes da educação básica (OAIGEN; BERNARD; SOUZA, 2013). Esses eventos são realizados no Brasil desde a década de 1960 com auge nos anos de 1990 (HARTMANN; ZIMMERMANN, 2009). Por envolver diversas áreas do conhecimento naturalmente favorece a interdisciplinaridade e a participação dos estudantes em debates atuais (SANTOS, 2012).

Atualmente nota-se uma estrutura verticalizada nas feiras científicas, os projetos mais bem avaliados em um evento local recebe credencial para participar em eventos a nível estadual, nacional e até mesmo internacional. Especificamente no Estado de Mato Grosso do Sul, as feiras científicas promovidas pela Rede Federal de Educação Profissional, Científica e Tecnológica, têm apresentado crescimento constante tanto no volume de projetos quanto nas afiliações. Este crescimento torna necessário o desenvolvimento de métodos eficientes para avaliação dos projetos.

Avaliação de projetos em feiras científicas pode ser classificada como Tradicional com as decisões tomadas por um grupo de avaliadores ou Participativas que envolve a comunidade em geral (MANCUSO, 2000). Independente do modo como é realizada a avaliação, julgamentos realizados por pessoas normalmente não são completamente racionais e estão sujeitos ao viés do avaliador (HARKER; VARGAS, 1987). 
Este estudo tem por objetivo apresentar um meio de avaliação de projetos em feiras científicas utilizando as abordagens Value-Focused Thinking (VFT) e Analytic Hierarchy Process (AHP) com medição absoluta (ratings). O VFT é utilizado para identificar critérios para julgamento dos projetos e o AHP com ratings com o objetivo de selecionar um projeto para receber credencial para uma Feira externa.

O restante deste artigo se organiza da seguinte maneira: A seção 2 contextualiza a respeito das feiras estudantis realizadas no Instituto Federal de Educação, Ciência e Tecnologia de Mato Grosso do Sul - Campus Corumbá (IFMS). Nesta seção também é apresentado de forma breve aspectos inerentes ao VFT e AHP. A seção 3 detalha proposta metodológica adotada e a seção 4 detalha a aplicação. Por fim, as conclusões do estudo são apresentadas na seção 5.

\section{REFERENCIAL TEÓRICO}

\subsection{Feiras de Ciência e Tecnologia do IFMS}

O IFMS promove todos os anos feiras de ciência e tecnologia em cada um dos 10 campus da instituição, os eventos ocorrem concomitante com a Semana Nacional de Ciência e Tecnologia - (SCT). Entre os maiores eventos do estado, a Feira de Ciência e Tecnologia do Pantanal em Corumbá (FECIPAN) reúne projetos de escolas das redes municipal, estadual e particular além dos projetos realizados pelos estudantes do próprio IFMS.

Os projetos mais bem avaliados na FECIPAN recebem credenciais para a Feira de Tecnologias, Engenharias e Ciências de Mato Grosso do Sul (FETEC) promovida pela Universidade Federal de Mato Grosso do Sul (UFMS) nível estadual sediada no município de Campo Grande, capital do estado. Outra credencial concedida é para a Feira Brasileira de Ciências e Engenharia (FEBRACE) promovida pela Universidade de São Paulo (USP) e considerado o maior evento do tipo no Brasil.

Outras premiações envolvem bolsas de iniciação científica do Conselho Nacional de Desenvolvimento Científico e Tecnológico (CNPq) pelo período de 1 ano, certificados por categoria e prêmios de parceiros. A primeira edição da FECIPAN foi realizada no ano de 2012 e contou com 48 projetos o auge ocorreu em 2016 com 151 projetos apresentados, para 2019 estão previstos mais de 100 em exposição.

Os projetos apresentados são das áreas: Ciências Biológicas e da Saúde; Exatas e da Terra; Humanas, Sociais Aplicadas e Linguística; Agrárias e Engenharias; e Multidisciplinar. A avaliação dos projetos é composta por um grupo multidisciplinar formado por docentes do IFMS da UFMS e por profissionais de outras instituições que desenvolvem pesquisas científicas na região. Todas essas informações estão disponíveis no portal do IFMS disponível em http://www.ifms.edu.br/. 


\subsection{Value-Focused Thinking (VFT)}

O VFT, introduzido por Ralph Keeney (KEENEY, 1992), propõe um meio criativo para identificar problemas de decisão, especificar objetivos, criar alternativas e critérios, atividades cruciais relacionadas ao processo de tomada de decisão (KEENEY, 1992). Desde sua introdução o VFT é estreitamente relacionado a modelos quantitativos de apoio as decisões como Multiple-Attribute Value Theory (MAVT), Multiple-Attribute Utility Theory (MAUT) e Multi-Criteria Decision Analysis (MCDA).

O VFT pode ser resumido em cinco etapas (SHENG; SIAU; NAH, 2010; TUHKALA et al., 2017), a seguir.

1. Definir valores;

2. Converter valores em objetivos;

3. Hierarquizar os objetivos;

4. Construir uma rede de objetivos meio-fim;

5. Definir critérios.

A lista de valores pode ser obtida por meio de perguntas tais como: "O que você quer? O que você valoriza? O que você deveria querer?" (KEENEY, 1994). Os valores abstratos são convertidos para objetivos tangíveis expressando-os no formato Verbo+Objeto. Um exemplo de um valor pode ser "Educação para todos para transformar uma nação" que pode ser convertido em objetivos tais como Universalizar o sistema de ensino; Melhorar a grade curricular; Qualificar os professores; Melhorar a infraestrutura etc.

Uma vez identificados os objetivos estes devem ser hierarquizados. Uma técnica conhecida como WITI Test (Why This Is Important? - Por que isso é importante?), permite identificar os objetivos meios, ou seja, aqueles que conduzem a determinado objetivo fundamental (KEENEY, 1996). Com os objetivos classificados é possível construir a rede de objetivos meio-fim e a partir dela pensar critérios e alternativas para alcançar os objetivos.

\subsection{Analytic Hierarchy Process (AHP) com Ratings}

Desenvolvido por Thomas L. Saaty em 1980, o AHP é um método de apoio multicritério a decisão, que propõe que o problema seja dividido em níveis hierárquicos, facilitando assim a compreensão e avaliação. Tem por vantagem também determinar uma ação global para cada alternativa, baseado no valor síntese atribuído pelo decisor (SILVA; BELDERRAIN; PANTOJA, 2010).

Segundo Saaty (SAATY, 2006) existem duas formas de realizar as comparações par a par utilizadas no método AHP, são elas: a) por meio de comparações em 
termos de medição relativa (do inglês, relative measurement); e, b) por meio de comparações em termos de medição absoluta (do inglês, absolute measurement). Na medição relativa, as alternativas são comparadas par a par à luz de um critério ou subcritério (se existir), ou seja todos os elementos são comparados entre si. Na medição absoluta (também conhecida como ratings) as alternativas são avaliadas de acordo com os níveis de intensidade (categorias) obtidos de cada atributo (critérios e subcritérios).

Ambas comparações (relativa e absoluta) são compostas em matrizes de comparação par a par e, em seguida, as prioridades locais e finais das alternativas são obtidas. O AHP com medição relativa pode ser usado quando o número de alternativas é relativamente pequeno $(<9)$. Caso o número de alternativas seja maior, é praticamente inviável compor as matrizes de comparação par a par, devido ao alto número de comparações a serem realizadas. Nesse caso, o AHP com medição absoluta resolve esse problema. Ou seja, os elementos no nível mais baixo da hierarquia (geralmente as alternativas) não são comparados entre si, mas são avaliados de acordo com as categorias dos critérios ou subcritérios que recebem. A Figura 1 apresenta a estrutura hierárquica do método AHP com ratings.

Figura 1: Estrutura hierárquica AHP com ratings

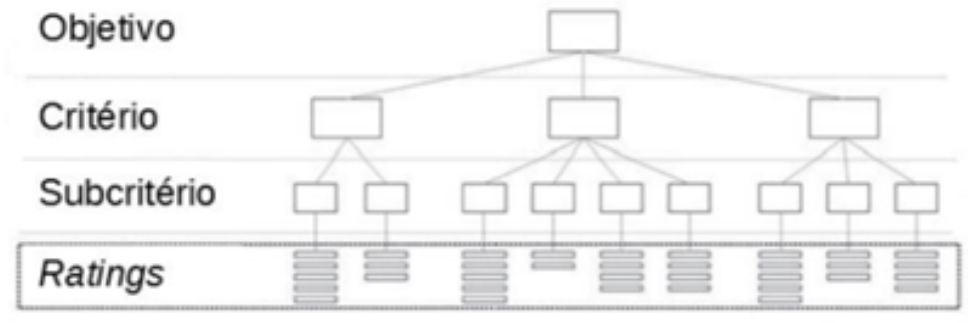

Fonte: (SILVA; BELDERRAIN; PANTOJA, 2010).

As categorias (ratings) de cada critério ou subcritério são comparadas entre si, usando o mesmo processo de comparação par a par do método AHP clássico, de forma a derivar os vetores de prioridades para as categorias. Os vetores de prioridades obtidos para as categorias são idealizados, ou seja, a melhor categoria recebe o valor igual a 1 e as outras receberam valores proporcionalmente menor (SAATY, 2006).

A síntese dos resultados, ou seja, as prioridades finais das alternativas são encontradas, somando-se os valores oriundos da multiplicação entre as prioridades de cada categoria e as prioridades globais dos critérios/subcritérios dessas categorias. As etapas para aplicação do método AHP com a abordagem ratings são: 1) formulação do problema, 2) julgamentos e 3) desenvolvimento algébrico (NASCIMENTO, 2010).

Na Etapa 1 (Formulação do Problema) define-se o objetivo do processo decisório, os critérios/subcritérios com suas respectivas categorias e as alternativas para a solução do problema. Posteriormente constrói-se a hierarquia e inserem-se as categorias nos critérios ou subcritérios. A hierarquia não possui no último nível as alternativas, porém estas são inseridas internamente na hierarquia. As alternativas 
são avaliadas selecionando-se a categoria apropriada de cada critério ou subcritério.

Na Etapa 2 (Julgamentos) as comparações par a par são entre os subcritérios (se existirem), entre os critérios e também entre as categorias de cada critério ou subcritério, de acordo com a Escala Fundamental de Saaty. Uma vez realizadas todas as comparações e verificada a consistência dos julgamentos são registrados os vetores de prioridades e as prioridades idealizadas das categorias (valores numéricos dos ratings).

Na Etapa 3 (Desenvolvimento Algébrico) são obtidas as prioridades locais e globais dos critérios e subcritérios. Por fim, obtêm-se as prioridades totais das alternativas, agregando as prioridades globais dos critérios ou subcritérios com os valores numéricos idealizados das categorias em cada critério ou subcritério. Maiores detalhes sobre as etapas e passos do método AHP com ratings (NASCIMENTO, 2010).

\section{PROPOSTA METODOLÓGICA}

A proposta metodológica é apresentada na Figura 2. Nela é possível identificar as atividades (fases) que foram desenvolvidas durante a aplicação do VFT e do método AHP com ratings para a seleção de projetos em feiras científicas estudantis.

Figura 2: Proposta Metodológica

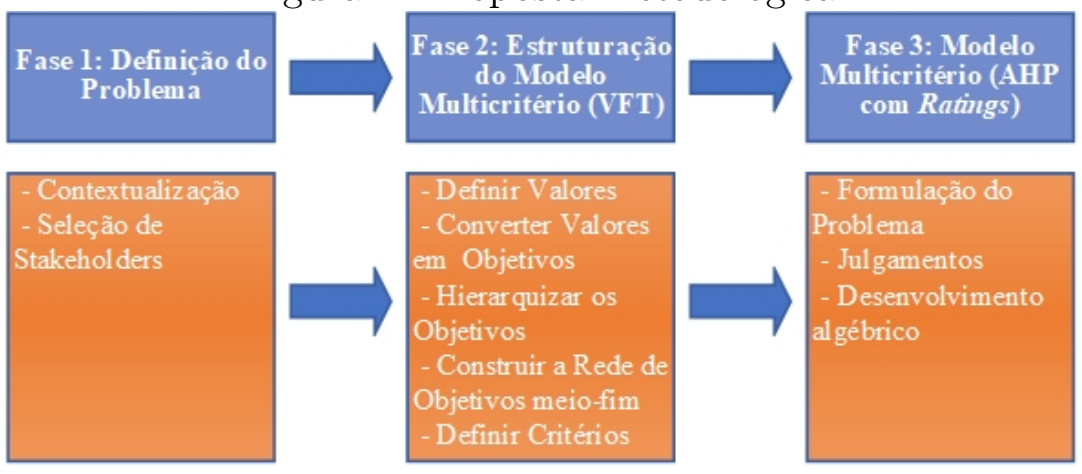

A Fase 1 "Definição do Problema" é formada pela contextualização do problema e a seleção de stakeholders. Os stakeholders foram identificados e classificados por meio do diagrama interesse e poder, destacado na Figura 3, onde os envolvidos com mais interesse e com mais poder são escolhidos para participar da intervenção (ACKERMANN; EDEN, 2011). 
Figura 3: Categorização de stakeholders.

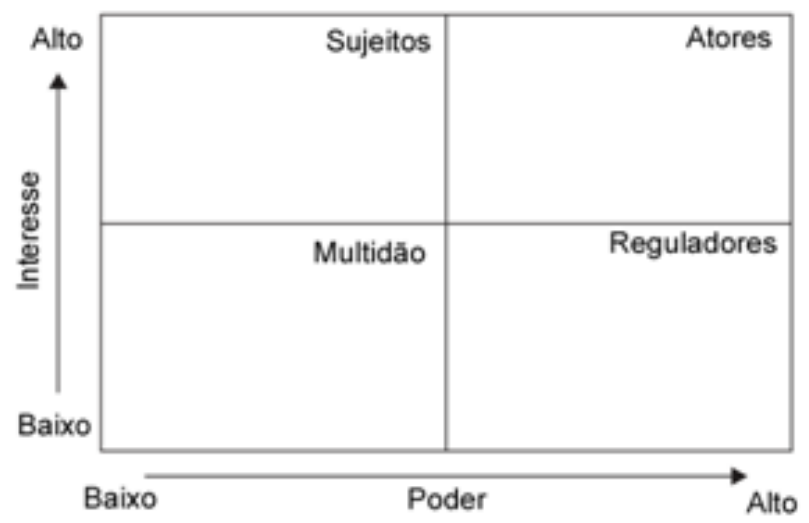

Fonte: Adaptado de (ACKERMANN; EDEN, 2011)

Na Fase 2 "Estruturação do Modelo Multicritério", foi aplicado o VFT como forma de identificar os objetivos e critérios para avaliação. Com os critérios definidos, construiu-se o modelo multicritério.

Na Fase 3 "Modelo Multicritério", foi aplicado o método AHP com ratings para a seleção dos projetos da feira científica estudantil.

\section{APLICAÇÃO DA PROPOSTA METODODÓGICA}

\subsection{FASE 1 - DEEFINIÇÃO DO PROBLEMA}

\subsubsection{Contextualização e Seleção de Stakeholders}

A FECIPAN é um evento tradicional no IFMS que ao longo dos anos cresceu em quantidade e qualidade dos projetos apresentados. Os melhores projetos recebem certificados, medalhas, troféus e credenciais para participar em outras feiras científicas a nível estadual e nacional. Os projetos são avaliados por profissionais convidados que estejam envolvidos com a pesquisa científica na região.

Uma das maiores dificuldades relacionadas com a avaliação dos projetos é a seleção de um (ou alguns) para participação em feira externa, uma vez que o intervalo de notas possíveis (6 a 10 com intervalo de 1) facilita a ocorrência de empates entre os projetos. Para lidar com a questão, ao invés de ampliar o intervalo de notas possíveis, iremos redesenhar o sistema de avaliação. Para esta atividade foram selecionados dois stakeholders seguindo a matriz de interesse e poder apresentada na Figura 4: 
Figura 4: Classificação dos Stakeholders

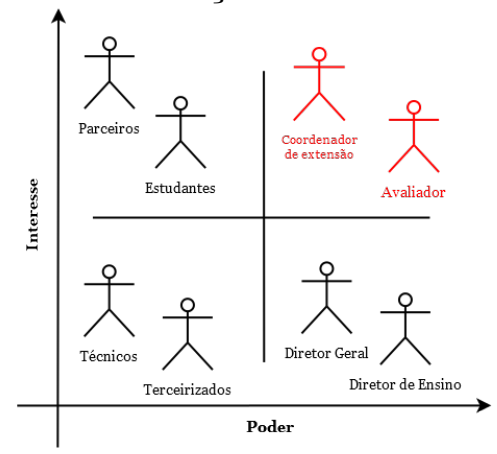

Os dois stakeholders selecionados são professores responsáveis pela organização da FECIPAN em algum momento ou que foram membros da comissão responsável pela avaliação dos projetos.

\subsection{FASE 2 - ESTRUTURAÇÃO DO MODELO MULTICRITÉRIO: VFT}

\subsubsection{Definir Valores}

Para este os dois professores foram entrevistados em conjunto. As seguintes perguntas foram utilizadas como guia para as entrevistas:

- Por que a FECIPAN é realizada?

- Por que os projetos da FECIPAN são avaliados?

- O que seria considerada uma situação ideal para os projetos da FECIPAN?

- Quais benefícios a avaliação dos projetos podem trazer?

- O que dificulta ou atrapalha o processo de avaliação?

Por meio de análise das respostas foram extraídos os valores dos envolvidos o que permitiu compreender as razões pela qual a FECIPAN é realizada e os objetivos da avaliação dos projetos. Os valores extraídos foram: divulgar as pesquisas científicas realizadas no IFMS, criar uma cultura na cidade, tornar o ambiente de ensino mais agradável e criar reputação do IFMS como instituição referência em pesquisa.

\subsubsection{Converter Valores em Objetivos}

Os valores foram convertidos para objetivos tangíveis. Conforme proposto por (KEENEY, 1996), utilizou-se no formato Verbo+Objeto. Esta técnica gerou uma "nuvem" de objetivos apresentados na Figura 5. 
Figura 5: Nuvem de Objetivos Gerados a Partir dos Valores

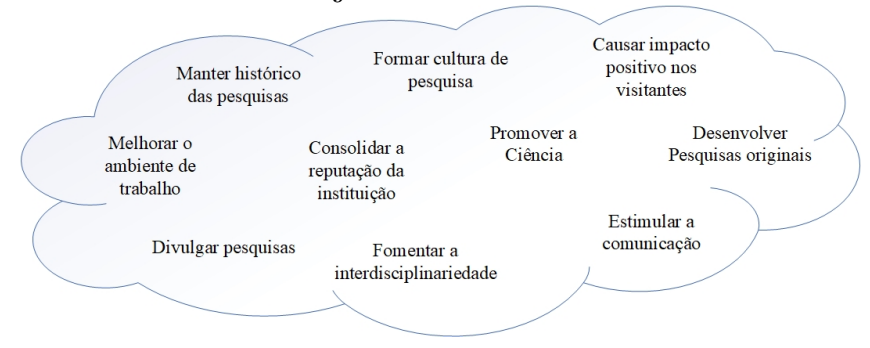

Nesta etapa também foi verificado se os objetivos atendem as seguintes propriedades: ser essencial, controlável, completo, mensurável, operacional, decomposto, não-redundante, conciso e entendível, conforme comentadas por (KEENEY, 1992).

\subsubsection{Hierarquizar os Objetivos}

Os objetivos apresentados na Figura 5 não apresentam nenhuma estrutura ou relação de dependência. Partindo do objetivo fundamental de: "Selecionar projeto para credenciamento em Feira externa", foi utilizado a técnica WITI - Why is that important para diferenciar os objetivos entre meios e fundamentais e estabelecer a hierarquia de objetivos conforme a Tabela 1.

Tabela 1: Hierarquia de Objetivos

\begin{tabular}{l}
\hline Selecionar projeto para credenciamento em Feira externa \\
\hline Manter histórico das pesquisas \\
Estimular a comunicação \\
Promover a Ciência \\
Fomentar a interdisciplinariedade \\
Divulgar pesquisas \\
Causar impacto positivo nos visitantes \\
Formar cultura de pesquisa \\
Melhorar o ambiente de trabalho \\
Desenvolver Pesquisas originais \\
Elaborar Protótipos \\
Consolidar a reputação da instituição \\
Fomentar a interdisciplinariedade
\end{tabular}

Após a hierarquização dos objetivos, construiu-se a rede de objetivos.

\subsubsection{Construir uma rede de objetivos meio-fim}

A Figura 6 apresenta a Rede de Objetivos, de forma a melhor visualizar seus relacionamentos. 
Figura 6: Rede de Objetivos meio-fim

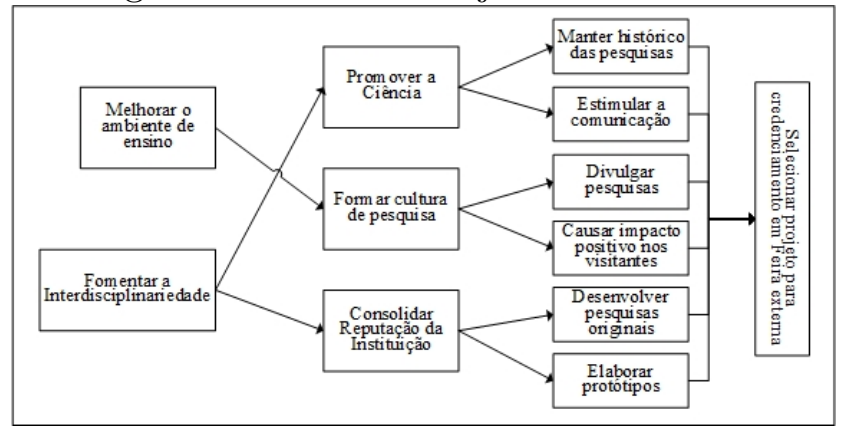

Os organizadores entendem que bons resultados em mostras científicas como a FEBRACE tem potencial de estimular os estudantes e contribuir na consolidação do IFMS como instituição indutora de desenvolvimento científico, social e tecnológico. A partir da rede de objetivos meio-fim foi possível extrair os critérios.

\subsubsection{Definir Critérios}

A análise da rede de objetivos meio-fim permitir aos stakeholders debater sobre quais os critérios devem ser utilizados para avaliar os projetos. Os 5 critérios identificados são apresentados na listagem a seguir:

- Apresentação Oral: A qualidade da apresentação dos estudantes durante a exposição e o domínio sobre seu projeto.

- Banner: A organização e clareza do Banner ou Pôster

- Diário de Bordo: Caderno de pesquisa do estudante onde realiza anotações relacionadas ao desenvolvimento do projeto.

- Maquete/Protótipo: A Maquete ou Protótipo pode ser um recurso físico como um experimento ou um software.

- Relatório de Pesquisa: Análogo ao Trabalho de Conclusão de Curso de graduação.

Identificados os critérios, procedeu-se a uma análise multicritério com a utilização do método AHP com ratings, de modo a selecionar projeto para o credenciamento em Feira externa.

\subsection{FASE 3 - MODELO MULTICRITÉRIO: AHP COM RATINGS}

O método AHP com ratings foi aplicado com o auxílio do software SuperDecisions (www.superdecisions.com). 


\subsubsection{Formulação do Problema}

Nesta etapa deve-se definir o objetivo do problema, os critérios com suas respectivas categorias (ratings) e as alternativas. Posteriormente constrói-se a hierarquia e inserem-se as categorias nos critérios.

O objetivo foi selecionar projeto para credenciamento em Feira externa. Os critérios surgiram dos objetivos fundamentais e meios do VFT. São eles: C1 - Apresentação Oral; C2 - Banner; C3 - Diário de Bordo; C4 - Maquete/Protótipo; e C5 - Relatório de Pesquisa. Considerou-se que todos os critérios do problema são independentes, como preconiza o método AHP.

As notas de 6,0 até 10,0 com intervalos de 1,0 foram definidas como categorias para os ratings para todos os critérios. Esse intervalo de notas foi decidido pelos stakeholders como mais adequado sugerindo que notas abaixo de 6,0 poderiam desestimular os estudantes a desenvolver novos projetos no futuro.

Os stakeholders decidiram adotar 10 projetos da área de Ciências Exatas e da Terra apresentados na FECIPAN de 2016 como alternativas no modelo. Tais projetos foram rotulados sequencialmente de CET01 - CET10.

A Tabela 2 apresenta as notas atribuídas em consenso pelos stakeholders para cada projeto de acordo com os ratings dos critérios estabelecidos.

Tabela 2: Notas (Ratings) atribuídas pelos stakeholders

\begin{tabular}{l|c|c|c|c|c}
\hline & C1 & C2 & C3 & C4 & C5 \\
& Apresentação & Banner & Diário & Protótipo & Relatório \\
\hline CET01 & 7.0 & 8.0 & 6.0 & 8.0 & 7.0 \\
\hline CET02 & 8.0 & 9.0 & 10.0 & 10.0 & 10.0 \\
\hline CET03 & 9.0 & 10.0 & 9.0 & 10.0 & 10.0 \\
\hline CET04 & 7.0 & 8.0 & 9.0 & 8.0 & 8.0 \\
\hline CET05 & 8.0 & 8.0 & 8.0 & 10.0 & 9.0 \\
\hline CET06 & 7.0 & 7.0 & 7.0 & 8.0 & 9.0 \\
\hline CET07 & 9.0 & 7.0 & 10.0 & 8.0 & 9.0 \\
\hline CET08 & 10.0 & 10.0 & 10.0 & 9.0 & 10.0 \\
\hline CET09 & 9.0 & 9.0 & 9.0 & 10.0 & 10.0 \\
\hline CET10 & 10.0 & 10.0 & 10.0 & 10.0 & 9.0 \\
\hline
\end{tabular}

A hierarquia do problema proposto está representada na Figura 7, bem com os ratings associados a cada critério. 
Figura 7: Estrutura Hierárquica do Problema

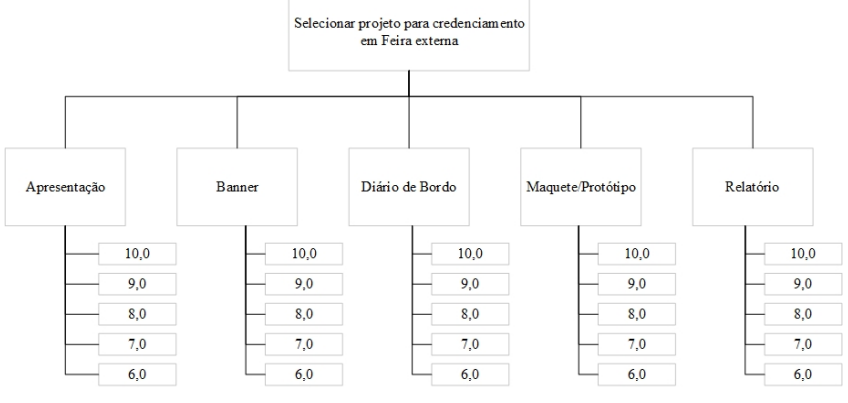

Na etapa seguinte, os stakeholders devem então iniciar o processo de julgamento, construindo as matrizes de comparação par a par necessárias para que, com os vetores prioridade calculados, possa se calcular as prioridades finais das alternativas.

\subsubsection{Julgamentos}

Nesta etapa são apresentadas as matrizes de comparação par a par para o presente problema.

Primeiramente os stakeholders fizeram o julgamento par a par para definir a importância de cada critério com relação aos demais e obter os pesos dos critérios (vetor de prioridade).

Todas as comparações par a par, inclusive as entre os ratings dos critérios, foram realizadas de acordo com a escala Fundamental de Saaty, de forma a obter-se as prioridades.

A Tabela 3 representa a matriz de comparação par a par dos critérios.

Tabela 3: Matriz de comparação par a par entre os Critérios à luz do Objetivo

\begin{tabular}{l|c|c|c|c|c|c}
\hline $\mathrm{RC}=0,09$ & $\mathrm{C} 1$ & $\mathrm{C} 2$ & $\mathrm{C} 3$ & $\mathrm{C} 4$ & $\mathrm{C} 5$ & Prioridade \\
\hline $\mathrm{C} 1$ - Apresentação & 1 & 3 & 5 & 7 & 5 & 0,487 \\
\hline $\mathrm{C} 2$ - Banner & $1 / 3$ & 1 & 3 & 6 & 5 & 0,265 \\
\hline C3 - Diário & $1 / 5$ & $1 / 3$ & 1 & 4 & 5 & 0,146 \\
\hline C4 - Protótipo & $1 / 7$ & $1 / 6$ & $1 / 4$ & 1 & 2 & 0,055 \\
\hline C5 - Relatório & $1 / 5$ & $1 / 5$ & $1 / 5$ & $1 / 2$ & 1 & 0,047 \\
\hline Razão de Consistência (RC)
\end{tabular}

Os stakeholders consideraram como principal fator da avaliação a apresentação oral dos estudantes que compõe projeto. O relatório foi considerado com a menor prioridade por não ser exigido, ou indispensável, para as mostras parceiras.

A Tabelas 4 refere-se à matriz de comparação dos ratings para todos os critérios.

Seguindo a escala de Saaty a leitura da matriz de comparação par a par dos ratings (Tabela 4) é dada da seguinte maneira: A categoria 10 recebe valor 9 quando 
comparada com a categoria 6 ou seja, a categoria 10 é extremamente mais importante que a categoria 6 .

Tabela 4: Comparações par a par dos ratings dos critérios C1 a C5

\begin{tabular}{c|c|c|c|c|c|c|c}
\hline $\mathrm{RC}=0,05$ & 6 & 7 & 8 & 9 & 10 & Prioridade & Prioridade idealizada \\
\hline 6 & 1 & $1 / 3$ & $1 / 5$ & $1 / 7$ & $1 / 9$ & 0,033 & 0,059 \\
\hline 7 & 3 & 1 & $1 / 3$ & $1 / 5$ & $1 / 7$ & 0,063 & 0,112 \\
\hline 8 & 5 & 3 & 1 & $1 / 3$ & $1 / 5$ & 0,129 & 0,237 \\
\hline 9 & 7 & 5 & 3 & 1 & $1 / 3$ & 0,261 & 0,498 \\
\hline 10 & 9 & 7 & 5 & 3 & 1 & 0,513 & 1,000 \\
\hline
\end{tabular}

Nas Tabelas 3 e 4 estão representadas também as colunas contendo o respectivo autovetor (prioridade) de cada matriz de comparação par a par.

Nesta aplicação, os stakeholders consideraram que todas as matrizes de comparação par a par dos ratings para os critérios C1 a C5 apresentam os mesmos julgamentos e consequentemente o mesmo vetor de prioridade.

De posse das matrizes de comparação par a par, realizou-se a consistência dos julgamentos. Todas as matrizes apresentaram RC (Razão de Consistência) menor que $10 \%$ (ou 0,1 ), indicando coerência dos julgamentos do decisor.

\subsubsection{Desenvolvimento algébrico}

A Figura 8 apresenta as prioridades globais dos critérios (primeira linha da tabela) e a classificação das alternativas (CET01 - CET10) nos ratings dos critérios, de acordo com as notas atribuídas em consenso pelos stakeholders para cada projeto (Tabela 2).

Figura 8: Classificação das alternativas nos ratings dos critérios C1 a C5

\begin{tabular}{|l|l|l|l|l|l|}
\hline Alternatives & $\begin{array}{l}\text { C1 Apresentaçăo } \\
(0.4870)\end{array}$ & $\begin{array}{l}\text { C2 Banner } \\
(0.2648)\end{array}$ & $\begin{array}{l}\text { C3 DiariodeBordo } \\
(0.1458)\end{array}$ & $\begin{array}{l}\text { C4 MaqueteProtótipo } \\
(0.0554)\end{array}$ & $\begin{array}{l}\text { C5 RelatóriodePesquisa } \\
(0.0470)\end{array}$ \\
\hline CET01 & 7.0 & 8.0 & 6.0 & 8.0 & 7.0 \\
CETO2 & 8.0 & 9.0 & 10.0 & 10.0 & 10.0 \\
CETO3 & 9.0 & 10.0 & 9.0 & 10.0 & 10.0 \\
CETO4 & 7.0 & 8.0 & 9.0 & 8.0 & 8.0 \\
CETO5 & 8.0 & 8.0 & 8.0 & 10.0 & 9.0 \\
CETO6 & 7.0 & 7.0 & 7.0 & 8.0 & 9.0 \\
CETO7 & 9.0 & 7.0 & 10.0 & 8.0 & 9.0 \\
CETO8 & 10.0 & 10.0 & 10.0 & 9.0 & 10.0 \\
CETO9 & 9.0 & 9.0 & 9.0 & 10.0 & 10.0 \\
CET10 & 10.0 & 10.0 & 10.0 & 10.0 & 9.0 \\
\hline
\end{tabular}

A Figura 9 apresenta a classificação das alternativas nos ratings dos critérios, utilizando os valores numéricos (valores em parênteses) correspondente das categorias (Tabela 4) e a prioridade total das alternativas.

A pontuação final para cada projeto é calculada do somatório dos produtos entre as prioridades globais dos critérios e os valores dos ratings (idealizados) para 
cada alternativa, obtendo-se a coluna "Totals", que normalizada apresenta a pontuação final, representada pela coluna "Priorities". Por exemplo, para o projeto CET01, tem-se: ProjetoCET01 $=(0,487 x 0,124)+(0,265 x 0,252)+(0,146 x 0,065)+$ $(0,055 x 0,252)+(0,047 x 0,124)=0,1560$.

Figura 9: Ratings idealizados dos critérios

\begin{tabular}{|l|l|l|l|l|l|l|l|}
\hline Alternatives & Priorities & Totals & $\begin{array}{l}\text { C1 Apresentą̧̧0 } \\
(0.4870)\end{array}$ & $\begin{array}{l}\text { C2 Banner } \\
(0.2648)\end{array}$ & $\begin{array}{l}\text { C3 DiariodeBordo } \\
(0.1458)\end{array}$ & $\begin{array}{l}\text { C4 MaqueteProtótipo } \\
(0.0554)\end{array}$ & $\begin{array}{l}\text { C5 RelatóriodePesquisa } \\
(0.0470)\end{array}$ \\
\hline CETO1 & 0.0312 & 0.1560 & $(0.1236) 7.0$ & $(0.2515) 8.0$ & $(0.0650) 6.0$ & $(0.2515) 8.0$ & $(0.1236) 7.0$ \\
CETO2 & 0.1010 & 0.5057 & $(0.2515) 8.0$ & $(0.5099) 9.0$ & $(1.0000) 10.0$ & $(1.0000) 10.0$ & $(1.0000) 10.0$ \\
CETO3 & 0.1378 & 0.6899 & $(0.5099) 9.0$ & $(1.0000) 10.0$ & $(0.5099) 9.0$ & $(1.0000) 10.0$ & $(1.0000) 10.0$ \\
CETO4 & 0.0453 & 0.2269 & $(0.1236) 7.0$ & $(0.2515) 8.0$ & $(0.5099) 9.0$ & $(0.2515) 8.0$ & $(0.2515) 8.0$ \\
CETO5 & 0.0609 & 0.3051 & $(0.2515) 8.0$ & $(0.2515) 8.0$ & $(0.2515) 8.0$ & $(1.0000) 10.0$ & $(0.5099) 9.0$ \\
CETO6 & 0.0297 & 0.1488 & $(0.1236) 7.0$ & $(0.1236) 7.0$ & $(0.1236) 7.0$ & $(0.2515) 8.0$ & $(0.5099) 9.0$ \\
CETO7 & 0.0928 & 0.4647 & $(0.5099) 9.0$ & $(0.1236) 7.0$ & $(1.0000) 10.0$ & $(0.2515) 8.0$ & $(0.5099) 9.0$ \\
CET08 & 0.1943 & 0.9728 & $(1.0000) 10.0$ & $(1.0000) 10.0$ & $(1.0000) 10.0$ & $(0.5099) 9.0$ & $(1.0000) 10.0$ \\
CETO9 & 0.1119 & 0.5601 & $(0.5099) 9.0$ & $(0.5099) 9.0$ & $(0.5099) 9.0$ & $(1.0000) 10.0$ & $(1.0000) 10.0$ \\
CET10 & 0.1951 & 0.9770 & $(1.0000) 10.0$ & $(1.0000) 10.0$ & $(1.0000) 10.0$ & $(1.0000) 10.0$ & $(0.5099) 9.0$ \\
\hline
\end{tabular}

No momento da análise dos resultados pelos stakeholders, o mesmo deve atentar para à coluna prioridade total (totals), pois quanto mais próximo de 1 este valor, melhor é o projeto. Por exemplo o CET10, apresentou um valor total de 0,977, pois apresenta nota máximo em quatro dos cincos critérios.

A Figura 10 apresenta as prioridades finais (na forma gráfica) para as alternativas.

Figura 10: Ranking final das alternativas

\begin{tabular}{|c|c|c|c|c|}
\hline Name & Graphic & Ideals & Normals & Raw \\
\hline CET01 & & 0.159692 & 0.031158 & 0.031158 \\
\hline CETO2 & & 0.517638 & 0.100998 & 0.100998 \\
\hline СЕTO3 & & 0.706182 & 0.137785 & 0.137785 \\
\hline CETO4 & & 0.232231 & 0.045311 & 0.045311 \\
\hline CET05 & & 0.312326 & 0.060939 & 0.060939 \\
\hline CETO6 & & 0.152350 & 0.029725 & 0.029725 \\
\hline CET07 & & 0.475691 & 0.092813 & 0.092813 \\
\hline СЕT08 & & 0.995793 & 0.194292 & 0.194292 \\
\hline СЕТО9 & & 0.573337 & 0.111865 & 0.111865 \\
\hline CET10 & & 1.000000 & 0.195113 & 0.195113 \\
\hline
\end{tabular}

Dentre os projetos analisados pelos stakeholders, observa-se que os quatro CETs que apresentam a maior proporção do ranking são os CETs 10, 08, 03 e 09.

O projeto mais bem avaliado foi CET10 com uma pequena margem para CET08, o que significa que em caso de impossibilidade de indicação do projeto CET10 para participação em feira externa, o projeto CET08 poderia ser indicado. A diferença que colocou CET10 a frente foi ter recebido nota máxima (10) no critério C4 (Maquete/Protótipo), que possui um peso um pouco maior do que o critério C5 (Relatório de Pesquisa).

Embora seja apenas um pequeno exemplo, a modelagem e avaliação dos projetos com AHP com ratings se mostra mais confiável especialmente por considerar as diferenças de relevância entre os critérios, tal tratamento não é nem mesmo minimamente tratado com avaliações envolvendo simples médias aritméticas. 


\section{CONCLUSÕES}

O objetivo deste estudo foi de apresentar um modelo alternativo para avaliação de projetos em feiras científicas. O modelo proposto combina a estruturação do problema com identificação do objetivo fundamental e critérios a serem considerados por meio do VFT com seleção dos projetos por meio do AHP com ratings.

Além de permitir identificar os critérios e definir a importância relativa na avaliação final de um projeto, a adoção do VFT possibilitou ainda tornar explícito o objetivo fundamental do processo de avaliação: Selecionar projeto para credenciamento em Feira externa. Além disso, deseja-se que o projeto selecionado consiga bons resultados na feira a qual foi credenciado. Por bons resultados compreende-se ficar entre os primeiros colocados ou receber credenciais para participar de outras mostras.

A adoção do AHP com ratings para avaliação dos projetos permitiu considerar a importância relativa dos critérios para a composição da avaliação final, algo que não era considerado anteriormente. Com a simples média entre as notas atribuídas pelos avaliadores com o mesmo peso entre os critérios ocorriam muitos empates ou era necessário um intervalo maior entre as notas, tornando o processo de avaliação bastante desafiador.

Além disso, o AHP com ratings permitiu apresentar aos stakeholders um processo decisório transparente, simples e de fácil implementação. O método também se destaca pela possibilidade de, caso necessário, retirar ou adicionar alternativas durante o processo decisório sem que haja dano na estruturação do problema, além de permitir uma quantidade maior de alternativas quando comparado com o AHP clássico.

A abordagem envolvendo métodos multicritérios se mostra mais eficiente que a adotada anteriormente para avaliação de projetos na FECIPAN. A metodologia adotada para este estudo pode ser utilizada em feiras científicas para além do processo de avaliação, como a seleção preliminar dos projetos que serão expostos, atividades complementares, seleção de palestrantes e mesmo de avaliadores, são alguns exemplos de atividades dentro das feiras científicas que podem ser abordadas com a metodologia proposta.

Considerar a aplicação do questionário a um número maior de stakeholders, com o objetivo de identificar outros fatores (critérios) que não foram evidenciados no presente estudo e descrever adequadamente as categorias (ratings) para estes outros critérios, são algumas das sugestões para trabalhos futuros.

\section{AGRADECIMENTOS}

Este trabalho foi parcialmente apoiado pelo Conselho Nacional de Desenvolvimento Científico e Tecnológico - CNPq. 


\section{REFERÊNCIAS BIBLIOGRÁFICAS}

ACKERMANN, F.; EDEN, C. Strategic management of stakeholders: Theory and practice. Long Range Planning, v. 44, n. 3, p. 179 - 196, 2011. ISSN 0024-6301. Disponível em: 〈http://www.sciencedirect.com/science/article/pii/ S0024630110000452〉. 6, 7

HARKER, P. T.; VARGAS, L. G. The theory of ratio scale estimation: Saaty's analytic hierarchy process. Management Science, v. 33, n. 11, p. 1383-1403, 1987. Disponível em:〈https://doi.org/10.1287/mnsc.33.11.1383〉. 2

HARTMANN, Â. M.; ZIMMERMANN, E. Feira de ciências: a interdisciplinaridade e a contextualização em produções de estudantes de ensino médio. Anais do VII Encontro Nacional de Pesquisa em Educação em Ciências-ENPEC; Florianópolis: ABRAPEC, 2009. 2

KEENEY, R. L. Value-focused thinking. [S.1.]: Harvard University Press, 1992. 4, 9

KEENEY, R. L. Using values in operations research. Operations Research, v. 42, n. 5, p. 793-813, 1994. Disponível em:〈https://doi.org/10.1287/opre.42.5.793〉. 4

KEENEY, R. L. Value-focused thinking: Identifying decision opportunities and creating alternatives. European Journal of Operational Research, v. 92, n. 3, p. 537 - 549, 1996. ISSN 0377-2217. Disponível em:〈http://www.sciencedirect.com/ science/article/pii/0377221796000045>. 4, 8

MANCUSO, R. Feiras de ciências: produção estudantil, avaliação, conseqüências. Contexto educativo: revista digital de investigación y nuevas tecnologías, Contexto Educativo, n. 6, p. 8, 2000. 2

NASCIMENTO, L. Aplicação do método AHP com as abordagens Ratings e BOCR: $O$ Projeto F-X2. Tese (Doutorado) - Tese de mestrado-Instituto Tecnológico de Aeronáutica. São José dos Campos, 2010. 5, 6

OAIGEN, E. R.; BERNARD, T.; SOUZA, C. A. Avaliação do evento feiras de ciências: aspectos científicos, educacionais, socioculturais e ambientais. Revista Destaques Acadêmicos, v. 5, n. 5, 2013. 2

SAATY, T. L. Rank from comparisons and from ratings in the analytic hierarchy/network processes. European Journal of Operational Research, v. 168, n. 2 , p. 557 - 570, 2006. ISSN 0377-2217. Feature Cluster on Mathematical Finance and Risk Management. Disponível em: 〈http://www.sciencedirect.com/science/ article/pii/S037722170400311X〉. 4, 5

SANTOS, A. B. dos. Feiras de ciência: um incentivo para desenvolvimento da cultura científica. Revista Ciência em Extensão, v. 8, n. 2, p. 155-166, 2012. 2

SHENG, H.; SIAU, K.; NAH, F. Understanding the values of mobile technology in education: A value-focused thinking approach. ACM SIGMIS Database, v. 41, p. 25-44, 05 2010. 4 
SILVA, A. C.; BELDERRAIN, M. C. N.; PANTOJA, F. C. M. Prioritization of r\&d projects in the aerospace sector: Ahp method with ratings. Journal of Aerospace Technology and Management, SciELO Brasil, v. 2, n. 3, p. 339-348, 2010. 4, 5

TUHKALA, A. et al. Identifying objectives for a learning space management system with value-focused thinking. In: SCITEPRESS SCIENCE AND TECHNOLOGY PUBLICATIONS. CSEDU 201\%: Proceedings of the 9th International Conference on Computer Supported Education. Vol. 1, ISBN 978-989-758-239-4. [S.l.], 2017. 4 